\title{
Structure of $\mathrm{SiO}$ masers in Orion-KL
}

\author{
Sheperd Doeleman, Colin Lonsdale, Paul Kondratko, Whitney Raas \\ MIT Haystack Observatory, Off Route 40, Westford, MA. 01886, USA \\ C. Read Predmore \\ University of Massachusetts, Dept. of Astronomy, 619 Lederle Graduate \\ Research Center, Amherst, MA 01003, USA
}

\begin{abstract}
.
The Orion-KL nebula is the closest (450pc) site of high-mass star formation and exhibits powerful outflows associated with protostars. It is also one of only three known star forming regions to exhibit $\mathrm{SiO}$ maser emission. Emission in three $\mathrm{SiO}$ maser transitions $(\mathrm{v}=1 \mathrm{~J}=1 \rightarrow 0, \mathrm{v}=1$ $\mathrm{J}=2 \rightarrow 1$, and $\mathrm{v}=2 \mathrm{~J}=1 \rightarrow 0$ ) imaged by VLBI exhibits an "X" morphology suggesting that the Orion masers form along the outlines of two opposing conical outflows to the NW and SE. At the center of this "X", VLA observations find emission from an HII region presumably associated with a young star whose wind drives the outflow. The SiO masers probably form along the interface between the stellar wind and surrounding parent cloud. We find that $\mathrm{SiO}$ maser emission from different transitions preferentially occurs at different radii from the central star implying that the masers are tracers for physical conditions in the wind-cloud interaction region. On the smallest scales, some individual maser features in each transition overlap both spatially and in velocity providing strong evidence that more than one transition can mase within the same volume of gas.
\end{abstract}

For many years, $\mathrm{SiO}$ masers in Orion-KL were thought to form in a protoplanetary disk around a young stellar object (YSO) (Barvainis 1984, Plambeck et al. 1990). Recent VLBI observations with both the Coordinated mm-VLBI Array (CMVA) and the NRAO VLBA reveal SiO maser structures which do not support a disk model. Instead, the maser emission arises in four main regions marking the arms of an " $\mathrm{X}$ " and filaments in each region extend radially towards a central point (Figs 1 and 2). Emission to the $\mathrm{N}$ and $\mathrm{W}$ is red-shifted relative to the systemic $\mathrm{SiO}$ velocity and to the $\mathrm{S}$ and $\mathrm{E}$, the emission is blue-shifted. This broad spatial and velocity structure suggests that the $\mathrm{SiO}$ masers form along the edges of a bi-polar outflow from a central star (Doeleman, Lonsdale, \& Pelkey 1999; Greenhill et al. 1998). VLA observations at $8.4 \mathrm{GHz}$ and $43 \mathrm{GHz}$ (Menten \& Reid 1995) place a continuum source with positive spectral index at the exact centroid of the $\mathrm{SiO}$ maser emission. This object, Source I, probably arises due to an ionized wind or HII region surrounding a deeply embedded YSO and may be powering a large scale outflow in the region seen in $\mathrm{CO}$ and shocked $\mathrm{H}_{2}$ (Chernin \& Wright 1982, Stolovy et al. 1998). The exact relationship between 
the outflow outlined by the $\mathrm{SiO}$ masers and larger scale water maser emission and the $\mathrm{CO}$ outflow is the subject of ongoing investigation.

A number of $\mathrm{SiO}$ maser transitions are observed in Orion-KL and their relative placement can provide important constraints on the maser pumping mechanism and the underlying physical conditions. Previous single-dish studies of $\mathrm{SiO}$ masers yield conflicting views on the apparent co-location of the $\mathrm{v}=1$ $\mathrm{J}=2 \rightarrow 1(86.2 \mathrm{GHz})$ and $\mathrm{J}=1 \rightarrow 0(43.1 \mathrm{GHz})$ transitions (McIntosh \& Predmore 1987, Barvainis and Predmore 1985). This is likely due to confusion caused by spectral blending of widely spaced maser features at similar velocities. Both collisional and radiative excitation mechanisms predict these $v=1$ transitions to be co-spatial (see Elitzur 1992). The $\mathrm{v}=2 \mathrm{~J}=1 \rightarrow 0$ transition is predicted to form closer to the central exciting source as it sits $\sim 1800 \mathrm{~K}$ higher above the ground state than either of the $v=1$ transitions (see also Humphreys this volume). In the context of AGB stars, Yi et al. (this volume) and Desmurs et al. (2000) find that the $v=2$ transition, on average, occurs closer to the central star.

Figs 1 and 2 show overlay maps of two transition pairs showing for the first time the relative positions of $\mathrm{SiO}$ maser emission around a YSO. As predicted, the $\mathrm{v}=2$ transition occurs closer to the central star. The $86.2 \mathrm{GHz} v=1$ transition, however, inhabits regions at larger radii from the center than does the 43.1 $\mathrm{GHz} \mathrm{v}=1$ line, a fact in stark contrast to expectations. The collisional models of Lockett \& Elitzur (1992) allow this type of offset for a narrow range of gas column densities, although their model uses an assumed spherical geometry around an evolved star, not a young star. If the Lockett \& Elitzur model is applicable, the $J=2 \rightarrow 1$ masers may trace a region of lower density in the interaction region between the stellar wind and the surrounding cloud. Radiative excitation models (Bujarrabal 1994) show little difference between intensities of the $v=1$ lines $(3 \mathrm{~mm}$ and $7 \mathrm{~mm})$ for varying physical conditions and may require a more restrictive parameter space to reproduce the observations.

In both Figures, we note that several maser features overlap precisely in both position and velocity, not only permitting robust positional registration between the images, but also demonstrating that multiple maser transitions can be excited within the same volume of gas. Combined with the spatial offsets between the transitions when viewed as an ensemble of maser spots, the complexity of the interplay between the maser pump and variations in the surrounding physical conditions becomes apparent. At this stage, detailed observations of $\mathrm{SiO}$ masers have outpaced theoretical explanations, and current VLBI results must be taken into account in new models. Only then can the extraordinary potential of these complex images as probes of circumstellar environments be fully realized.

\section{References}

Barvainis, R. E. 1984, ApJ, 279, 358

Barvainis, R. E. \& Predmore, C.R. 1985, ApJ, 288, 694

Bujarrabal, V. 1994, A\&A, 285, 953

Chernin, L.M. \& Wright, M.H. 1996, ApJ, 467, 676 
Desmurs, J. F., Bujarrabal, V., Colomer, F., \& Alcolea, J. 2000, A\&A, 360, $189 \mathrm{D}$

Doeleman, S.S., Lonsdale, C.J., \& Pelkey, S. 1999, ApJ, 510, L55

Greenhill, L.J., Gwinn, C.R., Schwartz, C., Moran, J.M., \& Diamond, P.J. 1998, Nature, 396, 650

Lockett, P. \& Elitzur M. 1992, ApJ, 399, 704

McIntosh, G.C. \& Predmore, C.R. 1993, ApJ, 404, L71

Menten, K.M. \& Reid, M.J. 1995, ApJ, 445, L157

Plambeck, R.L, Wright, M.C.H., \& Carlstrom, J.E. 1990, ApJ, 348, L65

Stolovy, S. R., Burton, M. G., Erickson, E. F., Kaufman, M. J., Chrysostomou, A., Young, E. T., Colgan, S. W. J., Axon, D. J., Thompson, R. I., Rieke, M. J., \& Schneider, G. 1998, ApJ, 492, L151

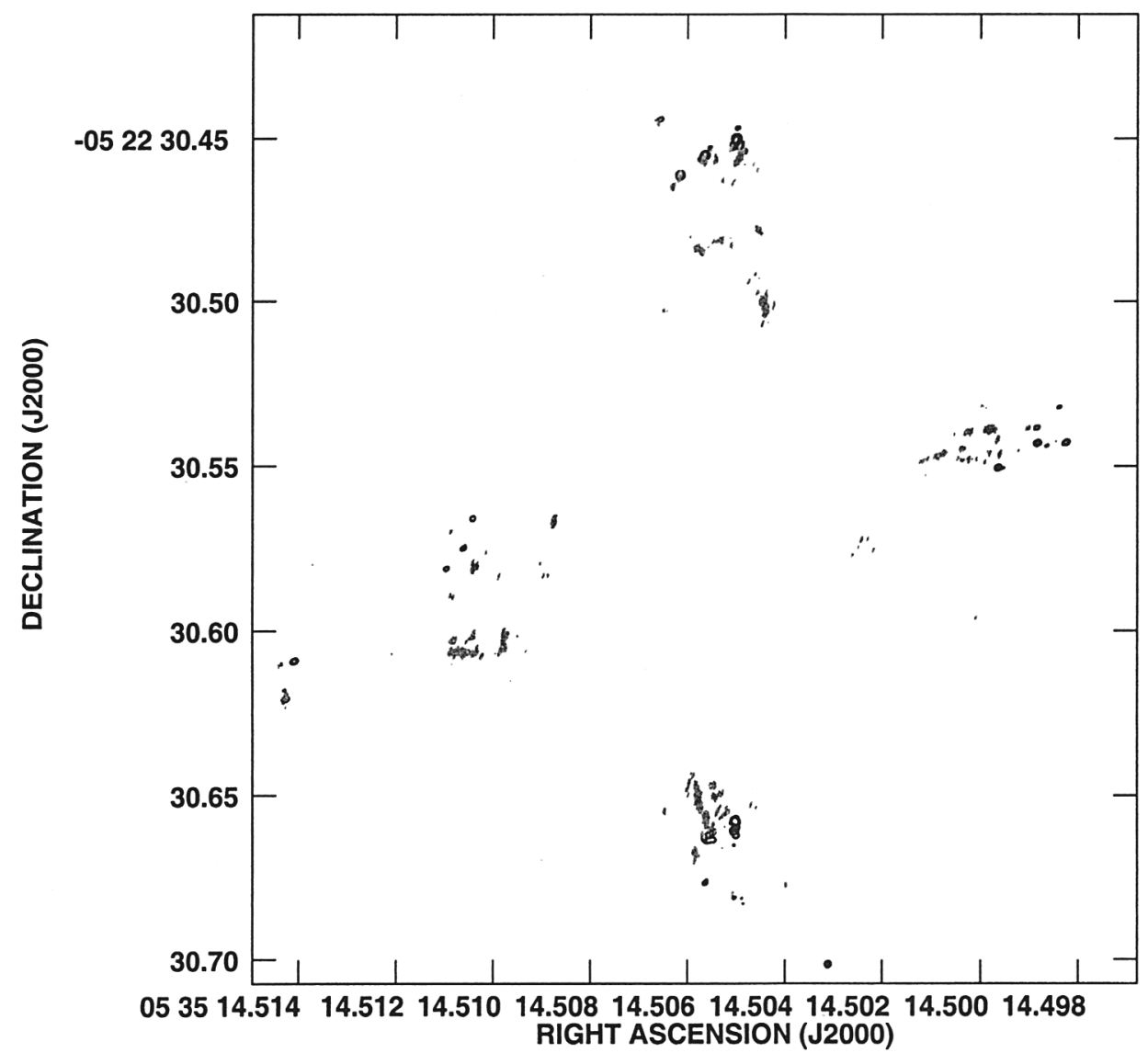

Figure 1. Overlay of $\mathrm{v}=1 \mathrm{~J}=2 \rightarrow 1$ (black) and $\mathrm{J}=1 \rightarrow 0$ (grey) transitions. Epoch is Oct 1999. 


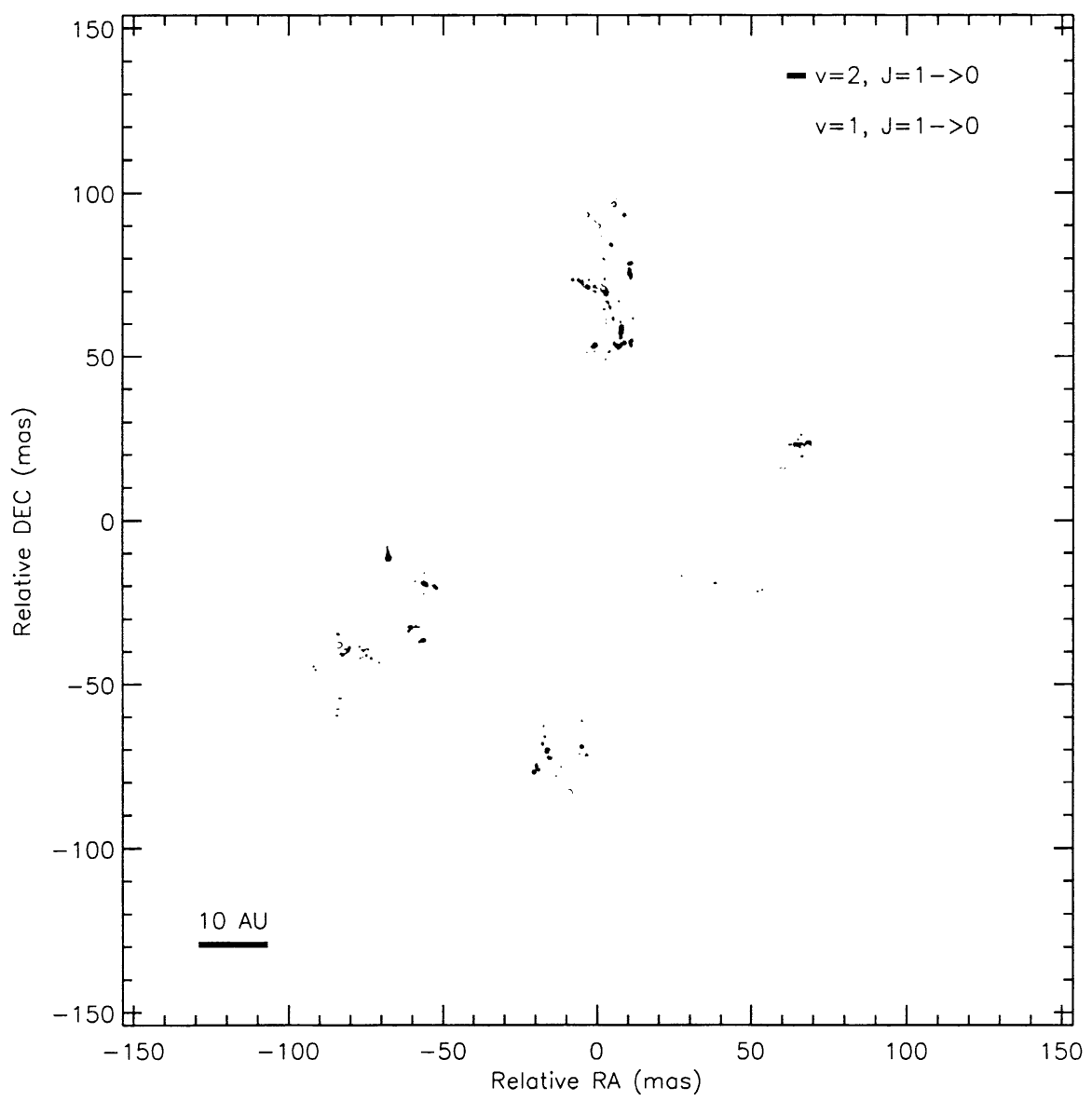

Figure 2. Overlay of $\mathrm{v}=2 \mathrm{~J}=1 \rightarrow 0$ (black) and $\mathrm{v}=1 \mathrm{~J}=1 \rightarrow 0$ (grey) transitions. Epoch is Oct 1997. 